\title{
Influence of Sport Motivation on Sport Mental Fatigue: Mediating Effect of Coping Style
}

\author{
Li Zhang ${ }^{1}$, Jingyi Xie ${ }^{1}$, Hao Zhang ${ }^{2 *}$ \\ ${ }^{1}$ Department of Physical Education, China University of Mining and Technology, Beijing 100083, P.R. \\ China \\ ${ }^{2}$ Department of Physical Education, Beijing University of Posts and Telecommunications, Beijing, 100876, \\ P.R. China \\ *Corresponding Author.
}

\begin{abstract}
:
In order to explore the relationship between sport motivation sport mental fatigue, as well as the mediating effect of coping style, a sample of 523 active athletes were recruited to complete sport motivation questionnaire, sport mental fatigue questionnaire and coping inventory for competitive sports. The result indicated that sport motivation negatively predicted the sport mental fatigue. Coping style partially mediated the relationship between sport motivation and sport mental fatigue. The mediating effect accounted for $65 \%$ of total effect. Sport motivation and coping style explain the $35 \%$ variation of sport mental fatigue. The harm effect of sport mental fatigue on sport motivation could be reduced while the coping style could be improved.
\end{abstract}

Keywords: Sport motivation, Sport mental fatigue, Coping style, Task-oriented coping, Emotion-oriented coping, Avoidance-oriented coping.

\section{INTRODUCTION}

Sport mental fatigue has become an important and difficult problem in the China Sport Science circles. It includes exhaustion of athletes' emotions and physical strength, reduction of their sense of accomplishment and negative evaluation. [1] Therefore, compared with sport physiological fatigue, the symptoms of sport mental fatigue are more complex. Sports-induced mental fatigue refers to that when athletes face endogenous and exogenous pressures, their psychological energy and physiological energy is continuously consumed and cannot be replenished in time. As a result, the psychological function declines and the original level of psychological activity cannot be maintained [2]. According to the above explanation, the 
problem of sport mental fatigue is the result of the joint action of endogenous and exogenous factors. In recent years, many scholars have made in-depth research on the mechanism of sport mental fatigue, and put forward the following theoretical models: sports commitment model, emotion-stress model, single identity development and external control model, self-determination theory and psychological capital theoretical model. Among them, the emotion-stress model has been widely recognized by scholars [3]. Studies have shown that the problem of sport mental fatigue refers to fatigue and burnout caused by monotonous and intense training and competition in long-term high-pressure environment [4]. Although sport mental fatigue is caused by multiple internal and external factors, the internal factors of individual are the key [5]. In recent years, researchers have gradually shifted from describing the phenomenon and nature of sport mental fatigue $\mathrm{n}$ to the influencing factors of sport mental fatigue, among which sport motivation is regarded as one of the important factors affecting sport mental fatigue.

The research on sport motivation started in 1970s [6]. It is considered as the internal driving force for athletes to participate in sports training, and it has the initial, directional and reinforcing effects on athletes' behavior [7]. Vallerand [8] divided sport motivation into three categories in his research, including external motivation, internal motivation and lack of motivation. Zhang Liwei [9] divided sport motivation into participation tendency and avoidance tendency. In recent years, the theory of self-determination has elaborated and divided the degree of sport motivation in detail. It regards sport motivation as a continuum of self-determination, and divides it into internal motivation, external motivation and lack of motivation according to the degree of self-determination [10].

Based on relevant literature at home and abroad, Raedeke et al pointed out that internal motivation is negatively correlated with mental fatigue, while lack of motivation is positively correlated with mental fatigue [11]. Zhang Liancheng and Zhang Liwei also confirmed this view in their research. Moreover, they also found that internal motivation and lack of motivation can effectively predict sport mental fatigue, while sport mental fatigue can effectively predict the participation tendency and avoidance tendency of sport motivation [12]. When Zeng Ming and Liu Wei put forward the relationship between sport mental fatigue and sport motivation, they found that athletes' lack of motivation for training would lead to increased mental fatigue [13]. Guo Yujiang found that there was a significant negative correlation between the total score of participation tendency of sport motivation and all dimensions and total scores of sport mental fatigue, and there was a significant positive correlation between the total score of avoidance motivation and all dimensions and total scores of sport mental fatigue [5]. Combined with the current research results, it can be found that the level of athletes' sport motivation will have a positive or negative impact on sport mental 
fatigue. Therefore, how to make athletes keep active and lasting sport motivation in the process of sports training and reduce sport mental fatigue caused by heavy load training is an urgent problem to be solved in current sports scientific research. Therefore, this study puts forward the hypothesis H1: sport motivation plays a significant role in negative prediction of sport mental fatigue.

In the process of literature review, the author thinks that coping style is an mediating variable worthy of consideration. Coping refers to the continuous cognitive and behavioral efforts to control the internal or external requirements after an individual evaluates that the internal or external requirements exceed his own resources [14]. According to the existing research results, researchers examine coping styles from the situational level, personal level and interactive level. Situation-based research holds that situation factors determine coping style. The research based on personal level holds that the differences in coping styles are caused by personality differences, while the research based on interactive level thinks that coping styles are a dynamic and cyclic process and are the interactive result of the interaction between the internal and external environment of individuals. The current research also tends to adopt the viewpoint of interaction [15]. As sport mental fatigue is the result of both endogenous and exogenous factors, coping style is the key factor to transfer the result of interaction between exogenous and endogenous factors to behavior [16]. Positive psychology suggests that setbacks and pressures faced by individuals will not necessarily lead to adverse consequences. If we are good at using all kinds of favorable resources and positive coping styles, we will be able to tide over the difficulties [17]. In recent years, some scholars in China have analyzed the mechanism of athletes' mental fatigue from the perspective of positive psychology, and put forward a "theoretical model of psychological capital", pointing out that adopting positive coping style is an important variable against mental fatigue [18]. Guo Yujiang found that the coping style of concentrated problem solving has a significant negative impact on athletes' mental fatigue. Avoidance and transcendence have significant positive effects on athletes' mental fatigue [19]. In their research, Li Wei, Mao Zhixiong and others found that positive coping style is an important factor to improve and relieve sport mental fatigue and promote athletes' training enthusiasm [20]. Wang Bin, Ye $\mathrm{Lu}$ and others put forward that changing athletes' coping style is the closer factor to changing athletes' mental fatigue. To relieve the symptoms of sport mental fatigue in a short time, it is an effective means to give priority to the intervention of coping style [21]. Lazarus put forward a theoretical model of cognition-motivation-relationship of emotion, which holds that coping style is an important mediating variable connecting training, competition results and stress psychophysiological response [14], and coping style should be regarded as an important mediating variable of competitive psychology and a factor of sport mental health. This intermediate variable plays a role in the process of circulation, and constantly influences the direction and intensity of 
psychological changes. Therefore, this study puts forward the hypothesis H2: coping style plays a mediating role between sport motivation and sport mental fatigue.

Taking coping style as an mediating variable, this study investigates athletes' sport motivation, sport mental fatigue and athletes' coping style, in order to verify the mediating effect of coping style in the process of sport motivation affecting sport mental fatigue, and to provide new ways and scientific references for stimulating and improving athletes' sport motivation and eliminating the negative effects of mental fatigue.

\section{RESEARCH METHODS}

\subsection{Subjects}

In this study, experiments were carried out in several sports teams in Chinese mainland. with the help of coaches of various sports, the outstanding athletes of various sports (all national first-class athletes or national elite athletes) were selected as subjects. In this study, a total of 547 questionnaires were distributed and all were collected on the spot. After eliminating the questionnaires with information omission, there were 523 valid questionnaires, with an effective recovery rate of $95.6 \%$. The subjects had an average age of $(20.25 \pm 2.08)$ years. There were 240 male professional athletes and 239 female professional athletes, 279 national first-class athletes and 200 national elite athletes. The tested sports involved basketball, football, volleyball, table tennis, track and field and badminton.

\subsection{Measuring Tools}

\subsubsection{Sport motivation scale}

Zhang Liwei's sport motivation scale [22] consists of two dimensions: participation tendency and avoidance tendency. Each dimension has 3 questions. The scale is composed of 6 questions with scores ranging from 0 to 24 . The Likert 5-level scoring method (0-4) is used to calculate the total score of athletes' sport motivation through accumulation. In this measurement, the alpha coefficient of the total scale is 0.841 ; the alpha coefficient of the participation tendency subscale is 0.826 , and the alpha coefficient of the avoidance tendency subscale is 0.881 . This shows that the scale has good reliability. The confirmatory factor analysis shows that $\chi^{2} / \mathrm{df}=2.69$. NNFI, CFI and IFI are $0.91,0.88$ and 0.85 respectively; RMSEA $=0.088$, less than 0.1 , indicating that the scale has good structural validity.

\subsubsection{Athletes' mental fatigue questionnaire}


The Exercise Burnout Questionnaire revised by Raedeke and Smith [23] has 3 dimensions, and each dimension has 5 questions. Likert 5-level scoring method is used for scoring. In the measurement, the alpha coefficient of total scale is 0.912 , among which the alpha coefficient of emotional/physical exhaustion subscale is 0.916, the alpha coefficient of sense of accomplishment reduction subscale is 0.850 , and the alpha coefficient of negative evaluation subscale is 0.889 . It shows that the scale has good reliability. In the measurement, the confirmatory factor analysis shows that $\chi^{2} / \mathrm{df}=2.75$, and NNFI CFI and IFI are 0.96, 0.98 and 0.98 respectively; RMSEA $=0.085$, less than 0.1 , indicating that the scale has good structural validity.

\subsubsection{Coping style questionnaire}

Coping Inventory for Competitive compiled by Gaudreau and Blondin [24] consists of three high-order dimensions: task-oriented coping, detachment-oriented coping and distraction-oriented coping. The questionnaire consists of 39 questions, which are scored by Likert 5-level scoring method. In this measurement, the alpha coefficient of total scale is 0.892 , among which the alpha coefficient of emotional/physical exhaustion subscale is 0.889 , the alpha coefficient of sense of accomplishment reduction subscale is 0.850 , and the alpha coefficient of negative evaluation subscale is 0.819 . It shows that the scale has good reliability. In the measurement, the confirmatory factor analysis shows that $\chi^{2} / \mathrm{df}=2.85$, and NNFI, CFI and IFI are $0.97,0.94$ and 0.93 respectively. RMSEA $=0.083$, less than 0.1 , indicating that the scale has good structural validity.

\subsection{Measurement and Data Statistics}

Before the test, the investigators in this study contacted the coaches or managers of sports teams in advance. With the approval of coaches, athletes and the management departments involved, the test fees were paid to athletes. Researchers chose to distribute questionnaires uniformly to athletes before and after they attended cultural courses in classroom or meeting room or collective meetings, so as to ensure a quiet and comfortable measuring environment, and the whole process of questionnaire filling and answering was completed accompanied by the main examiners. Before answering the questionnaire, the researcher clearly informed the athletes that the questionnaire was filled in anonymously and only for scientific research, and the information obtained would be kept strictly confidential. The whole test lasted for about 15 minutes. The questionnaire were checked and confirmed by researchers and then collected on site. 
This study is exploratory. To reduce the error of self-rating and ensure the preciseness and authenticity of the research data, this study adopts the following methods: first, considering that the subjects may have some concerns when they see the questionnaire of sport mental fatigue and cannot express their true thoughts, before the questionnaire is distributed, the investigators of this study will hide the title of the questionnaire. Secondly, in the guide part of the questionnaire, the respondents are indicated in conspicuous fonts that the questionnaire is anonymous. It is promised that the information they fill in would only be used for academic research, not for any other commercial purposes, and that the information they filled in would be kept strictly confidential. Third, the investigators make explanation to athletes the purpose of this survey, namely collecting data for university researchers to carry out academic research, and encourage them to answer honestly as much as possible. The data are tested by SPSS23.0 for reliability and validity, correlation analysis and hierarchical regression analysis.

\section{RESEARCH RESULTS}

\subsection{Correlation between Sport Motivation, Sport Mental Fatigue and Coping Style}

To test the correlation among sport motivation, sport mental fatigue and coping style, Pearson correlation analysis was used in this study. The results show that (Table I): First, there is a significant positive correlation between sport motivation and task-oriented coping ( $\mathrm{p}<$ 0.01), while there is a significant negative correlation between sport motivation and distraction-oriented coping and detachment-oriented coping $(\mathrm{p}<0.01)$. Secondly, there is a significant negative correlation between sport motivation and sport mental fatigue $(\mathrm{p}<0.01)$. Finally, there is a significant negative correlation between task-oriented sports and mental fatigue coping ( $\mathrm{p}<0.01$ ), and a significant positive correlation between distraction-oriented coping, distraction-oriented coping and exercise-oriented mental fatigue $(\mathrm{p}<0.01)$.

\section{Table I. Table of correlation}

\begin{tabular}{|l|l|l|l|l|l|}
\hline & 1 & 2 & 3 & 4 & 5 \\
\hline 1. SPORT MOTIVATION & 1 & & & & \\
\hline 2. TASK-ORIENTED COPING & $0.486 * *$ & 1 & & & \\
\hline 3. EMOTION-ORIENTED COPING & $-0.538^{* *}$ & $-0.360^{* *}$ & 1 & & \\
\hline 4. AVOIDANCE-ORIENTED COPING & $-0.554 * *$ & $-0.338^{* *}$ & $0.304 * *$ & 1 & \\
\hline 5. ATHLETE BURNOUT & $-0.604 * *$ & $-0.551^{* *}$ & $0.515^{* *}$ & $0.522^{* *}$ & 1 \\
\hline
\end{tabular}

Note: $*$ indicates $\mathrm{p}<0.05 * *$ indicates $\mathrm{p}<0.01, * * *$ indicates $\mathrm{p}<0.01$ (the same below).

3.2 The Mediating Effect of Coping Style on Sport Motivation and Sport Mental Fatigue 
To test the mediating effect of coping style between sport motivation and sport mental fatigue, this study adopted the mediating effect test procedure (Table II). Step 1: Test the predictive effect of independent variables on dependent variables. After controlling demographic variables such as age, sex and sports grade, the results show (Table II) that sport motivation plays a significant role in negative prediction of sport mental fatigue $(\beta-0.604, \mathrm{p}<$ 0.05), so the hypothesis $\mathrm{H} 1$ is verified. Step 2: Test the predictive effect of independent variables on mediating variables. According to results (Table II), exercise motivation can positively and negatively predict task-oriented coping $(\beta 0.486, \mathrm{p}<0.001)$, and plays a significant role in negative prediction of distraction-oriented coping and detachment-oriented coping $(\beta-0.538, \mathrm{P}<0.01 ; \beta-0.554, \mathrm{p}<0.001)$. Step 3: Test the predictive effect of mediating variables on dependent variables. According to results (Table II), task-oriented coping plays a significant role in negative prediction of sport mental fatigue $(\beta-0.286, \mathrm{p}<0.001)$, while distraction-oriented coping and detachment-oriented coping play a significant role in positive prediction of sport mental fatigue $(\beta 0.226, \mathrm{P}<0.001 ; \beta 0.239, \mathrm{p}<0.001)$. In step 3 , after controlling the demographic variables such as age, sex, sports grade and coping style, the predictive coefficient of sport motivation on sport mental fatigue increased from -0.604 to -0.211 , but it was still at a significant level. Therefore, it can be seen that task-oriented coping, distraction-oriented coping and detachment-oriented coping have some mediating effects between sport mental fatigue and sport motivation, so the research hypothesis $\mathrm{H} 2$ is verified. According to the results in the following table (Table II), the effect decomposition table (Table III) can be obtained. According to the effect decomposition table (Table III), the total effect of sport motivation on sport mental fatigue is -0.604 , the direct effect is -0.211 , and the indirect effect is -0.393 . The mediating effect of coping style accounts for $65 \%$ of the total effect. Sport motivation and coping style together explain $35 \%$ of the variation of sport mental fatigue.

Table II. Mediating effect test results

\begin{tabular}{|c|c|c|c|c|c|c|c|c|c|c|}
\hline & \multicolumn{2}{|c|}{$\begin{array}{l}\text { ATHLETE } \\
\text { BURNOU } \\
\mathrm{T}\end{array}$} & \multicolumn{2}{|c|}{$\begin{array}{l}\text { TASK-ORIE } \\
\text { NTED } \\
\text { COPING }\end{array}$} & \multicolumn{2}{|c|}{$\begin{array}{l}\text { EMOTION-ORI } \\
\text { ENTED } \\
\text { COPING }\end{array}$} & \multicolumn{2}{|c|}{$\begin{array}{l}\text { AVOIDANCE-O } \\
\text { RIENTED } \\
\text { COPING }\end{array}$} & \multicolumn{2}{|c|}{$\begin{array}{l}\text { ATHLETE } \\
\text { BURNOU } \\
\mathrm{T}\end{array}$} \\
\hline & \multicolumn{2}{|c|}{ M1 } & \multicolumn{2}{|c|}{ M2 } & \multicolumn{2}{|c|}{ M3 } & \multicolumn{2}{|c|}{ M4 } & \multicolumn{2}{|c|}{ M1 } \\
\hline & B & SE & B & SE & $\mathrm{B}$ & SE & $\mathrm{B}$ & SE & B & SE \\
\hline GENDER & $\begin{array}{l}-0.06 \\
7\end{array}$ & $\begin{array}{l}0.06 \\
1\end{array}$ & 0.031 & 0.048 & $-0.112 *$ & 0.010 & -0.072 & 0.071 & 0.000 & $\begin{array}{l}0.02 \\
9\end{array}$ \\
\hline AGE & 0.158 & $\begin{array}{l}0.01 \\
0\end{array}$ & 0.077 & 0.009 & $-0.011^{*}$ & 0.014 & 0.140 & 0.019 & $\begin{array}{l}0.116 \\
* *\end{array}$ & $\begin{array}{l}0.06 \\
6\end{array}$ \\
\hline SPORT LEVEL & -0.13 & 0.05 & 0.051 & 0.040 & 0.077 & 0.019 & 0.098 & 0.156 & -0.08 & 0.01 \\
\hline
\end{tabular}


Article History: Received: 10 May 2021 Revised: 20 June 2021 Accepted: 18 July 2021 Publication: 31 August 2021

\begin{tabular}{|c|c|c|c|c|c|c|c|c|c|c|}
\hline & 9* & 2 & & & & & & & $1 *$ & 7 \\
\hline SPORT & $\begin{array}{l}-0.60 \\
\end{array}$ & 0.13 & $0.486 *$ & 0.123 & $-0.538^{* *}$ & 0.187 & $-0.554 * * *$ & 0.053 & -0.21 & 0.16 \\
\hline MOTIVATION & $4 *$ & 4 & $* *$ & & & & & & $1 * *$ & 5 \\
\hline $\begin{array}{l}\text { TASK-ORIENTE } \\
\text { D COPING }\end{array}$ & & & & & & & & & $\begin{array}{l}-0.28 \\
6 * * *\end{array}$ & $\begin{array}{l}0.06 \\
5\end{array}$ \\
\hline $\begin{array}{l}\text { EMOTION-ORIE } \\
\text { NTED COPING }\end{array}$ & & & & & & & & & $\begin{array}{l}0.226 \\
* * *\end{array}$ & \begin{tabular}{|l|}
0.04 \\
2
\end{tabular} \\
\hline $\begin{array}{l}\text { AVOIDANCE-O } \\
\text { RIENTED } \\
\text { COPING }\end{array}$ & & & & & & & & & $\begin{array}{l}0.239 \\
* * *\end{array}$ & \\
\hline R2 & \multicolumn{2}{|l|}{0.365} & \multicolumn{2}{|l|}{0.236} & \multicolumn{2}{|l|}{0.29} & \multicolumn{2}{|l|}{0.307} & \multicolumn{2}{|l|}{0.527} \\
\hline $\mathrm{F}$ & \multicolumn{2}{|c|}{$\begin{array}{l}125.506^{* *} \\
*\end{array}$} & \multicolumn{2}{|c|}{$67.337 * * *$} & \multicolumn{2}{|c|}{$89.033^{* * *}$} & \multicolumn{2}{|l|}{$96.553 * * *$} & \multicolumn{2}{|c|}{$59.775^{* * *}$} \\
\hline
\end{tabular}

Table III. Effect resolution table

\begin{tabular}{|l|l|l|}
\hline IMPACT PATH & $\begin{array}{l}\text { STANDARDIZATIO } \\
\text { N EFFEC }\end{array}$ & $\begin{array}{l}\text { PERCEN } \\
\text { TAGE }\end{array}$ \\
\hline $\begin{array}{l}\text { SPORT MOTIVATION } \rightarrow \text { ATHLETE BURNOUT DIRECT } \\
\text { EFFECT }\end{array}$ & -0.211 & $34.9 \%$ \\
\hline $\begin{array}{l}\text { SPORT MOTIVATION } \rightarrow \text { TASK-ORIENTED COPING } \rightarrow-0.139 \\
\text { ATHLETE BURNOUT }\end{array}$ & $23.0 \%$ \\
\hline $\begin{array}{l}\text { SPORT MOTIVATION } \rightarrow \text { EMOTION-ORIENTED } \\
\text { COPING } \rightarrow \text { ATHLETE BURNOUT }\end{array}$ & 20.122 \\
\hline $\begin{array}{l}\text { SPORT MOTIVATION } \rightarrow \text { AVOIDANCE-ORIENTED } \\
\text { COPING } \rightarrow \text { ATHLETE BURNOUT }\end{array}$ & $21.9 \%$ \\
\hline $\begin{array}{l}\text { SPORT MOTIVATION } \rightarrow \text { ATHLETE BURNOUT TOTAL } \\
\text { EFFECT }\end{array}$ & -0.604 \\
\hline
\end{tabular}

\section{DISCUSSION AND ANALYSIS}

\subsection{The Direct Effect of Sport Motivation}

Firstly, this study investigates the predictive effect of sport motivation on sport mental fatigue. After investigation, it is found that sport motivation plays a significant role in negative prediction of sport mental fatigue, and there is a negative correlation between sport motivation and sport mental fatigue. The research results of Guo Yujiang [5], Zeng Ming [13], Wang Bin and others [21] all support this view. 
In recent years, in the field of sports psychology, researchers have gradually shifted from describing the phenomenon and nature of sports psychology to the influencing factors of sport mental fatigue, among which motivation is one of the important factors affecting sport mental fatigue. As a new motivation theory, self-determination theory emphasizes the degree of self-determination of human behavior, regards motivation as a continuum according to the degree of self-determination, and holds that social environment can enhance human internal motivation, promote internalization of external motivation and ensure healthy growth of individuals by supporting the satisfaction of three basic psychological needs: autonomy, competence and relationship.

Self-determination theory can reflect the subjective purpose of athletes participating in certain sports training, and the different positions of self-determination degree in the continuum reflect the level of autonomy and self-involvement of athletes in the process of sports training [10]. This is directly related to the level of fun, training satisfaction and mental fatigue that athletes can experience in the process of sports training. When athletes have higher sport motivation for a certain sport, their mental fatigue degree is light. However, when athletes participate in a certain sport because of compelling reasons or just external rewards, and they have no strong driving force to participate in the sport, they will have higher sport mental fatigue. The input model designed by Schmidt et al also supports the self-determination theory [25]. According to the model, if an athlete regards participating in a certain sport as a kind of enjoyment, he will face sports training with a more positive attitude and continue to invest enthusiasm to maintain his sports career. On the contrary, if he thinks that a certain sport makes him feel restrained, he is more likely to have burnout, and it will be difficult for him to persist in sports training for a long time. Therefore, athletes' motivation is closely related to their attitude towards sports training. The results of this study show that sport motivation plays a significant role in negative prediction of sport mental fatigue, and there is a negative correlation between sport motivation and sport mental fatigue, which confirms the relevant research at home and abroad.

From the research on the relationship between sport motivation and sport mental fatigue, it can be found that higher sport motivation can inhibit sport mental fatigue. Although sport motivation has strong individual subjectivity, it is easily disturbed by changes in internal and external environment. This suggests that intervention measures should be taken to improve athletes' sport motivation, which may be an effective way to alleviate and eliminate sport mental fatigue.

\subsection{The Mediating Effect of Coping Style}


Previous studies have shown that sport motivation has a significant negative effect on sport mental fatigue, but its mechanism is still unclear. Zeng Ming and Liu Wei discussed the relationship between them with mental toughness as a mediating variable, and found that mental toughness played a partial mediating role [13]. Guo Yujiang regarded time management as a mediating variable, and found that time management also plays a partial mediating role in sport motivation and sport mental fatigue [5]. In this study, coping style is used as a mediating variable to observe its mediating effect on sport motivation and sport mental fatigue, and some meaningful results are obtained. It can be seen from the test results of mediating model and the proportion of influence effects (Table III) that although sport motivation has a direct impact on sport mental fatigue, its direct effect is $34.9 \%$. The indirect effect of sport motivation on sport mental fatigue through coping style is $65 \%$. In other words, indirect effect is the main way that sport motivation affects sport mental fatigue.

In this study, coping styles mainly include three types, namely, task-oriented coping, distraction-oriented coping and detachment-oriented coping. Task-oriented coping refers to the coping strategies that athletes focus on meeting coaches' requirements and completing sports training and competition tasks. Distraction-oriented coping refers to the coping strategies in which athletes shift their attention from the completion of tasks to factors unrelated to tasks. Disengagement-oriented coping refers to strategies for athletes to disengage their attention from the struggle process of goal pursuit. Previous studies have shown that the above three coping styles have opposite potency. Task-oriented coping can positively predict results, such as goal achievement, competition satisfaction and positive emotions, while distraction-oriented coping and detachment-oriented coping can negatively predict results [21, 26]. For athletes, task-oriented coping is a more active and effective coping style, which can bring ideal stress adaptation results to individuals. The results of this study confirm the above viewpoint: task-oriented coping has a significant positive predictive effect on sport mental fatigue, while distraction-oriented coping and detachment-oriented coping have a significant negative predictive effect on sport mental fatigue. Therefore, coping style is an important factor affecting sport mental fatigue. Moreover, there are significant differences in the utility value of adopting different coping styles. This is consistent with the research findings of Lazarus [14] and Wang Bin et al. [21]. Liu Xun found that athletes' sport mental fatigue can be alleviated through the mediating variable of positive coping style [16]. Some scholars pointed out that the coping style adopted by individuals belongs to the category of personality traits, which will be influenced by the individual's own initiative, the acquired environment, education and training and other factors [27]. Therefore, this study suggests that targeted and planned guidance and training can be adopted to cultivate positive individual coping styles. 


\subsection{Practical Implications}

This study has some practical implications for the intervention practice of sport mental fatigue. First of all, in sports training, we should pay more attention to and guide the problem of sport motivation. Among it, athletes' sport motivation can be improved mainly by stimulating the material or spiritual level. Material incentives mainly include increasing monetary rewards, etc. This incentive means has limited time to maintain athletes' sport motivation, and it needs to be continuously overweight to temporarily maintain athletes' sport motivation. Therefore, compared with material incentives, spiritual incentives are the key to ensure the continuity of sport motivation. Suggestions for spiritual motivation in this study include: 1) Scientific establishment of short-term training objectives. Many athletes have the ideal of winning glory for their motherland. They usually set ambitious long-term goals for themselves, but often neglect the formulation of short-term goals. The disadvantage of setting long-term goals only is that it is difficult to gain results in a short time, and it is easy for athletes to lose motivation and enthusiasm. Therefore, short-term goals should be set scientifically, so that athletes can see their own progress and drive the promotion of sport motivation. 2) Increasing the freshness of training. Long-term acceptance of the same coach's guidance, long-term use of the same training plan and long-term training competition with fixed teammates will make athletes feel tired of training. Therefore, it is possible to increase the freshness of sports training by rotating the competent coaches, changing the training methods and contents, and regularly organizing training competitions with other sports teams. 3) Increasing positive evaluation. Many coaches have strict requirements on athletes in the training process, and they often focus on suppression teaching, which easily dampens athletes' self-esteem and self-confidence and suppresses their sport motivation. Therefore, positive evaluation of athletes' training performance should be added in training to strengthen and consolidate athletes' sport motivation.

Secondly, we should also strengthen the correct guidance of athletes' coping styles. In the process of sports training, we should strengthen task-oriented coping and reduce the occurrence probability of distraction-oriented coping and detachment-oriented coping. Studies have shown that when coaches explain the mechanism of different coping styles to athletes in detail and ask athletes to consciously choose coping styles, athletes' coping styles will become more flexible, and there will be no stubborn and unconscious reuse of ineffective coping styles [28]. Studies have found that many athletes cannot use effective coping styles even though they realize that their coping styles are ineffective and know other more effective coping styles, such as task-oriented coping styles. In this regard, behavior guidance and behavior shaping from coaches are very necessary intervention methods [29]. In the process of implementation, the effective coping style is taken as the theme of learning and strengthening according to 
behaviorism. Athletes are assisted to record or consciously record the occurrence frequency of positive coping by themselves during training.

When the frequency of using positive coping style increases significantly, coaches give appropriate material incentives or verbal praise to strengthen athletes' positive coping style. This study shows that this recording method can promote the strengthening of positive coping style. At present, coping effectiveness training, COPE training, and cognitive-emotional stress management training are the most extensive coping interventions [30]. Using these training methods can significantly improve athletes' coping effectiveness, self-efficacy and subjective performance, and reduce athletes' sport mental fatigue. Some studies have found that athletes can not only effectively cope with sport mental fatigue and stress events in training competitions, but also apply these learned coping strategies to daily life and study [21]. In the future, researchers can further compare and analyze the above intervention programs, test the actual results of different training programs, distinguish the advantages and disadvantages of each intervention training program, and develop more systematic and targeted intervention training programs.

\section{RESEARCH CONCLUSION}

To sum up, this study draws the following conclusions: 1) According to the correlation analysis, there is a significant positive correlation between sport motivation and task-oriented coping, and a significant negative correlation between sport motivation and distraction-oriented coping and detachment-oriented coping. There is a significant negative correlation between sport motivation and sport mental fatigue and between task-oriented sports and mental fatigue coping. There is a significant positive correlation between distraction-oriented coping and detachment-oriented coping and sports-oriented mental fatigue. 2) Sport motivation plays a significant role in negative prediction of sport mental fatigue. 3) Coping style plays a partial mediating effect between sport mental fatigue and sport motivation.

\section{ACKNOWLEDGEMENTS}

This work was supported by the Fundamental Research Funds for the Central Universities (2020SKTY01).

\section{REFERENCES}

[1] Zhang LC, Zhang LW, Yang HY, Liu Y (2012) Research progress on measurement and evaluation of sport mental fatigue. Chinese Journal of Sports Medicine 31(02):180-185. 
[2] Zhang LW, Lin L, Zhao FL (2006) Sport mental fatigue: nature, causes, diagnosis and control. China Sport Science 11:49-56+74.

[3] Zhang TT, Zhi EL (2018) The relationship between stress perception and exercise-induced mental fatigue: a mediating model with regulation. Journal of Shanghai University of Sport 42(01):104-110.

[4] Zhang LW (2010) Seven directions of psychological research: taking sport mental fatigue as an example. China Sport Science 30(10):3-12.

[5] Guo YJ (2015) The relationship between athletes' sport motivation and mental fatigue: the mediating effect of time management. Journal of Shenyang Sport University 34(05):43-47.

[6] Yan X (2019) Theme evolution analysis of motivation research in international sports psychology based on co-word analysis. Journal of Xi'an Physical Education University 36(06):743-747+768.

[7] Zhang LW, Mao ZX (2007) Sports Psychology. Beijing: Higher Education Press: 24.

[8] Vallerand RJ (1997) Toward A Hierarchical Model of Intrinsic and Extrinsic Motivation. Advances in Experimental Social Psychology 29(08):271-360.

[9] Zhang LW (2001) Factor structure, self-evaluation and attention characteristics of pre-competition emotion. Beijing: Beijing Sport University Press:107-108.

[10] Sun GX, Zhang LW (2013) The influence of self-determination motivation on athletes' mental fatigue: evidence from horizontal and vertical research. China Sport Science 33(07):21-28.

[11] Raedeke TD, Smith AL (2001) Development and Preliminary Validation of an Athlete Burnout Measure. J Sport Exerc Psychol 23(4):281-306.

[12] Zhang LC, Zhang LW, Liu JH, Liu H (2010) The relationship between athletes' mental fatigue and sport motivation. Journal of Beijing Sport University 33(11):74-76.

[13] Zeng M, Liu W (2013) The relationship between athletes' mental fatigue and sport motivation: the mediating effect of mental toughness. Journal of Wuhan Institute of Physical Education 47(11):76-80.

[14] Lazarus RS (2013) Fifty Years of the Research and Theory of R.S. Lazarus: An Analysis of Historical and Perennial Issues. Lawrence Erlbaum Associates.

[15] Wu M, Wang B (2016) Athletes' coping with stress: concept, theoretical model, influencing factors and prospect. Journal of Harbin Sport University 34(01):17-23.

[16] Liu X (2019) The influence of stress on athletes' mental fatigue: a mediating adjustment model. Journal of Shenyang Sport University 38(03):100-105.

[17] Seligman MEP, Csikszentmihalyi M (2015) Positive psychology: An introduction. American Psychologist 55(1):5-14.

[18] Zhang LC, Li SH, Liu Y (2014) Review on the theoretical research progress of sport mental fatigue. Journal of Physical Education 21(01):98-103.

[19] Guo YJ (2017) The relationship between coping style and sport mental fatigue: the moderating effect of two kinds of social support. Journal of Beijing Sport University 40(12):64-71.

[20] Li W, Mao ZX, Zhou ZG (2005) Current situation and prospect of Chinese sports coping research. China Sport Science 02:82-85+94.

[21] Wang B, Ye L, Wu M, Feng T, Peng X (2014) The influence of mental toughness on athletes' burnout: the mediating effect of coping style. Journal of Wuhan Institute of Physical Education 48(08):63-68. 
[22] Zhang LW, Mao ZX (2004) Scientific manual of psychological scale commonly used in China Sport Science. Beijing: Beijing sports university press:33-34.

[23] Raedeke Thomas D, Smith Alan L (2001) Development and Preliminary Validation of an Athlete Burnout Measure 23(4):281-306.

[24] Gaudreau P, Blondin JP (2002) Development of a questionnaire for the assessment of coping strategies employed by athletes in competitive sport settings 3(1):1-34.

[25] Schmidt GW, Stein GL (2010) Sport commitment: A model integrating enjoyment, dropout, and burnout. Journal of Sport \& Exercise Psychology 8(3):254-265.

[26] Schellenberg BJI, Gaudreau P, Crocker PRE (2013) Passion and coping: relationships with changes in burnout and goal attainment in collegiate volleyball players. J Sport Exerc Psychol 35(3):270-280.

[27] Guo L (2013) The characteristics of college athletes' coping style and its relationship with self-awareness-a typical correlation study. Journal of Beijing Sport University 36(02):84-87.

[28] Smith RE (1986) Toward a cognitive-affective model of athletic burnout.

[29] (2008) Influence of perceived coaching behaviors on burnout and competitive anxiety in female college athletes. Journal of Applied Sport Psychology.

[30] Ye L, Wang B, Wu M, Liu ZJ, Dong LS (2016) The influence of social support on athletes' input: the sequence mediation between psychological resilience and coping style. Journal of Beijing Sport University 39(07):75-82. 